\title{
EFEITO DA VARIABILIDADE DO ARMAZENAMENTO DE ÁGUA NO SOLO NA QUALIDADE DA IRRIGAÇÃO POR ASPERSÃO ${ }^{1}$
}

\author{
Carlos Ricardo Fietz ${ }^{2}$, Marcos Vinícius Folegatti ${ }^{3}$, Sidney Rosa Vieira ${ }^{4}$ \& José Antônio Frizzone ${ }^{3}$
}

\begin{abstract}
RESUMO
O objetivo deste trabalho foi avaliar a influência da variabilidade do armazenamento de água no solo, na qualidade da irrigação por aspersão. Com base em curvas características e lâminas de irrigação de 144 pontos amostrais de uma grade de $5 \times 5 \mathrm{~m}$, utilizando-se técnicas geoestatísticas, confeccionouse uma malha 1 x $1 \mathrm{~m}$ de 3096 pontos. Com os valores de lâmina real necessária e de lâmina aplicada determinou-se, para cada ponto da malha, a eficiência de armazenamento, a drenagem profunda e o grau de adequação. Esses parâmetros também foram avaliados pelo modelo linear, que considera a lâmina necessária constante em toda a área. A variabilidade do armazenamento de água no solo não influenciou, de forma relevante, a eficiência de armazenamento nem os índices de drenagem profunda, enquanto o modelo linear foi pouco sensível em diferenciar áreas deficientes e adequadamente irrigadas.
\end{abstract}

Palavras-chave: qualidade da irrigação, lâmina real necessária, variabilidade espacial

\section{INFLUENCE OF SOIL WATER STORAGE VARIABILITY ON THE QUALITY OF SPRINKLE IRRIGATION}

\begin{abstract}
The aim of this research was to evaluate the influence of soil water storage variability upon the quality of sprinkle irrigation systems. Based on the soil water retention curves and water depth measurements on 144 points of a $5 \times 5 \mathrm{~m}$ grid, and using geostatistics techniques, a $1 \times 1 \mathrm{~m}$ grid was calculated, totalizing 3096 points. With the values of required and applied depths, the storage efficiency, the deep drainage and irrigation adequacy were determined for each of these points. These parameters were also evaluated using the linear model, which considers an average required depth. The variability of soil water storage did not influence significantly the storage efficiency and the deep drainage indices. The linear model showed low sensitivity at distinguishing deficient and adequately irrigated areas.
\end{abstract}

Key words: irrigation quality, required depth, spatial variability

\footnotetext{
${ }^{1}$ Parte da tese apresentada à Universidade de São Paulo (ESALQ/USP) pelo primeiro autor para obtenção do título de Doutor em Agronomia

${ }^{2}$ Engenheiro Agrônomo, Dr. EMBRAPA-CPAO, CP 661, CEP 79804 - 970, Dourados, MS, E-mail fietz@cpao.embrapa.br

${ }^{3}$ Engenheiro Agrônomo, Professor Doutor, Departamento de Engenharia Rural da Escola Superior de Agricultura "Luiz de Queiroz", CP 09, CEP 13418 - 900, Piracicaba, SP

${ }^{4}$ Engenheiro Agrônomo, Dr., Seção de Conservação do Solo, Instituto Agronômico, CP 28, CEP 13001 - 970, Campinas, SP
} 


\section{INTRODUÇÃO}

No dimensionamento e manejo da irrigação, faz-se necessário o conhecimento de parâmetros que expressem e quantifiquem a qualidade da operação. Basicamente, além da uniformidade de aplicação, a qualidade de uma irrigação pode ser definida por dois outros parâmetros de desempenho: pela eficiência e pelo grau de adequação.

A eficiência de distribuição e de armazenagem estão entre os parâmetros mais utilizados na avaliação da performance da irrigação. A eficiência de distribuição mede a quantidade de água armazenada na zona radicular em relação à infiltrada, enquanto a eficiência de armazenagem indica a adequação do reabastecimento na profundidade efetiva.

O grau de adequação é definido como a fração da área que recebe a quantidade de água capaz de manter a qualidade do produto e a produtividade vegetal no nível econômico desejado pelo agricultor (Frizzone, 1998). Como essa definição é muito ampla e requer a especificação da cultura, do solo e das condições de mercado, o grau de adequação é geralmente definido de forma simplificada, como a porcentagem da área que recebe, no mínimo, a lâmina real necessária (Cuenca, 1989).

A distribuição da água na zona radicular das culturas pode ser analisada de diversas maneiras; uma forma muito utilizada é se ajustar a relação lâmina de água infiltrada e área irrigada a um modelo matemático, como o normal (Walker, 1979) ou o linear (Karmeli, 1978) e a partir dos mesmos avaliar a qualidade da irrigação. A utilização de modelos matemáticos tem uma série de vantagens, sendo a principal a possibilidade de simular o desempenho de um sistema avaliado com outro manejo de irrigação.

Segundo Souza \& Ribeiro (1984) o modelo linear destaca-se entre os demais por ser simples, acurado e de fácil aplicação; esse modelo relaciona a curva de frequiência acumulada da lâmina aplicada e a fração da área irrigada, ambas adimensionalizadas, através de uma regressão linear.

Algumas simplificações são adotadas na análise da qualidade da irrigação. Na prática, toda irrigação apresenta certo grau de desuniformidade, que resulta em áreas irrigadas com excesso ou com déficit; na área sem déficit hídrico, a quantidade de água que se infiltra no solo em excesso é considerada perdida por percolação, mas na área com déficit, considera-se que toda a água infiltrada é armazenada na zona radicular; além disso, assume-se que a lâmina real necessária é constante em toda a área irrigada, desprezando-se a variabilidade espacial do armazenamento de água no solo.

O objetivo deste trabalho foi avaliar a influência da variabilidade do armazenamento de água no solo, na qualidade da irrigação por aspersão.

\section{MATERIAL E MÉTODOS}

O ensaio foi realizado no Centro de Pesquisa Agropecuária do Oeste (EMBRAPA/CPAO) em Dourados, MS, cujas coordenadas geográficas são: $22^{\circ} 14^{\prime}$ de latitude sul, $54^{\circ} 49^{\prime}$ de longitude oeste e altitude média de $452 \mathrm{~m}$. O clima da região é o Cwa de Köppen (mesotérmico úmido, com verão chuvoso e inverno seco).
O local do ensaio, cultivado com trigo (Triticum aestivum, L.) possui solo classificado como Latossolo Roxo álico epieutrófico. $\mathrm{Na}$ área experimental, que tinha $3600 \mathrm{~m}^{2}(40 \mathrm{~m}$ por $90 \mathrm{~m})$ foi demarcada uma malha retangular de 8 colunas e 18 linhas, com espaçamento regular de $5 \mathrm{~m}$ (144 pontos amostrais); em cada ponto foram coletadas, na profundidade de $0,15 \mathrm{~m}$, amostras de solo com estrutura indeformada de $0,055 \mathrm{~m}$ de diâmetro e $0,040 \mathrm{~m}$ de altura. Essas amostras foram submetidas às tensões de 10; 33; 50; 100; 500 e $1500 \mathrm{kPa}$, utilizando-se câmara de pressão de Richards (EMBRAPA, 1997).

A área do ensaio era dotada de um sistema de irrigação por aspersão autopropelido. Os carreadores desse sistema tinham espaçamento de $50 \mathrm{~m}$ e foram locados na direção paralela e a $5 \mathrm{~m}$ de distância dos lados maiores da área experimental. O padrão de aplicação de água do sistema foi determinado com base em um ensaio, no qual as lâminas foram coletadas em cada ponto amostral, através de pluviômetros instalados a $0,60 \mathrm{~m}$ da superfície.

O modelo de van Genuchten (1980) ajustado aos seis pares umidade-tensão das curvas características, foi utilizado para determinar os valores de umidade do solo na tensão $60 \mathrm{kPa}$, recomendados como umidade do solo no momento da irrigação (Comissão Centro-Sul Brasileira de Pesquisa de Trigo, 1995). O momento da irrigação foi definido através de tensiômetros instalados a $0,15 \mathrm{~m}$ de profundidade.

A análise dos valores discrepantes foi realizada seguindo o procedimento apresentado por Libardi et al. (1996) ou seja: (i) avaliação estatística e (ii) avaliação visual. Considerou-se, como prováveis dados discrepantes, valores menores que a diferença do quartil inferior e 1,5 vez a amplitude interquartílica ou maior que a soma do quartil superior com 1,5 vez a amplitude interquartílica. A decisão de se excluir dados foi tomada após se confrontar os candidatos com seus vizinhos mais próximos em gráficos de distribuição espacial apresentados por Fietz (1998).

A existência de estrutura de variabilidade espacial foi avaliada através da análise variográfica dos dados. A seleção do modelo teórico e dos parâmetros de ajuste de cada semivariograma foi realizada pela técnica de validação cruzada (Vieira et al., 1983; Isaaks \& Srivastava, 1989).

Os semivariogramas experimentais dos dados de umidade nas tensões 10 e $60 \mathrm{kPa}$ e de lâmina aplicada, foram utilizados para se confeccionar, através da técnica de krigagem, malhas de 35 colunas e 85 linhas separadas por 1 m (3096 pontos); a lâmina real necessária à cultura do trigo foi determinada a partir desses valores de umidade, considerando-se $0,30 \mathrm{~m}$ como profundidade efetiva do sistema radicular da cultura (Doorenbos \& Kassan, 1979).

No ajuste dos semivariogramas, na validação cruzada e nas interpolações por krigagem, utilizaram-se os softwares desenvolvidos por Vieira et al. (1983) e o programa Geo-EAS (Englund \& Sparks, 1988).

A qualidade da irrigação foi avaliada através da simulação de diversos valores de lâmina aplicada em cada ponto da malha 1 x $1 \mathrm{~m}$, considerando-se duas situações:

a) armazenamento da água no solo variável: determinou-se o valor do excesso ou déficit hídrico em cada ponto da malha. $\mathrm{O}$ grau de adequação foi calculado pela relação de lâmina aplicada e lâmina real necessária de cada quadrícula de $1 \mathrm{~m}^{2}$. A eficiência de armazenamento (Es) e a drenagem profunda (Dp) foram 
determinadas, respectivamente, pelas equações (1) e (2):

$$
\begin{gathered}
\mathrm{Es}=\frac{\mathrm{Vs}}{\mathrm{Vr}} \\
\mathrm{Dp}=\frac{\mathrm{Vp}}{(\mathrm{Vp}+\mathrm{Vs})}=1-\mathrm{Ed}
\end{gathered}
$$

em que Vs é a quantidade de água armazenada na profundidade efetiva, Vr é a quantidade de água requerida na irrigação, Vp é a água perdida por drenagem profunda e Ed é a eficiência de distribuição.

b) armazenamento da água no solo constante: considerou-se a lâmina real necessária constante em toda a área e igual à média dos 3096 pontos; os parâmetros grau de adequação, eficiência de armazenagem e drenagem profunda, foram avaliados pelo modelo linear (Karmeli, 1978) utilizando-se as expressões apresentadas por Souza \& Ribeiro (1984) e Leme (1986).

\section{RESULTADOS E DISCUSSÃO}

Com base no critério de Libardi et al. (1996) três curvas de retenção foram excluídas, pois possuíam mais de dois valores de umidade, considerados pela estatística como discrepantes e também apresentaram diferenças consideráveis de seus vizinhos. Vários dados de lâmina aplicada foram considerados, pela estatística, atípicos, mas nenhum foi excluído, pois apresentavam coerência com a sua localização, conforme foi verificado na avaliação visual; portanto, a grande variabilidade e a amplitude dos valores de lâmina aplicada (Tabela 1) podem ser atribuídas à distribuição irregular do sistema autopropelido.

Tabela 1. Estatísticas descritivas de lâmina necessária e de lâmina aplicada na malha 1 x $1 \mathrm{~m}$ (3096 pontos estimados)

\begin{tabular}{lcc}
\hline Estatística & $\begin{array}{c}\text { Lâmina real } \\
\text { necessária }(\mathrm{mm})\end{array}$ & $\begin{array}{c}\text { Lâmina aplicada } \\
(\mathrm{mm})\end{array}$ \\
\hline Média & 9,8 & 11,0 \\
Mediana & 9,7 & 11,3 \\
Mínimo & 5,4 & 0,4 \\
Máximo & 19,5 & 17,1 \\
Amplitude & 14,1 & 16,7 \\
Quartil inferior & 9,0 & 9,2 \\
Quartil superior & 10,2 & 13,8 \\
Amplitude interquartílica & 1,2 & 4,6 \\
Desvio-padrão & 1,54 & 3,73 \\
Coeficiente de variação & 0,16 & 0,34 \\
Assimetria & 1,72 & $-0,64$ \\
Curtose & 6,90 & 0,04 \\
D $^{1}$ & 0,14 & 0,07 \\
CUC & - & 73,0 \\
\hline 1 Valores de máxima divergência do teste Kolmogorov-Smirnov para a distribuição normal. O nível em \\
5\% de significância é de 0,02
\end{tabular}

Os valores de umidade nas tensões 10 e 60 kPa e de lâmina aplicada apresentaram dependência espacial. Os semivariogramas experimentais de umidade (Fig. 1A) ajustaramse satisfatoriamente a modelos exponenciais, com alcance de 22,0 m (Tabela 2) e, assim como obtido por Gonçalves et al. (1996) apresentaram valores diferenciados de efeito pepita e de patamar. Para fins práticos, como no caso do planejamento de novas amostragens, procurou-se ajustar, com o mesmo alcance, os modelos de semivariograma dos dados de umidade. O semivariograma experimental dos dados de lâmina aplicada (Fig. 1B) foi ajustado a um modelo gaussiano com alcance de $28,0 \mathrm{~m}$ e valores de efeito pepita e de patamar de, respectivamente, 0,5 e 20,8. Deve-se ressaltar que o modelo gaussiano é utilizado para ajustar fenômenos extremamente contínuos (Isaaks \& Srivastava, 1989) o que evidencia o alto grau de dependência espacial dos dados de lâmina aplicada (Tabela 2).

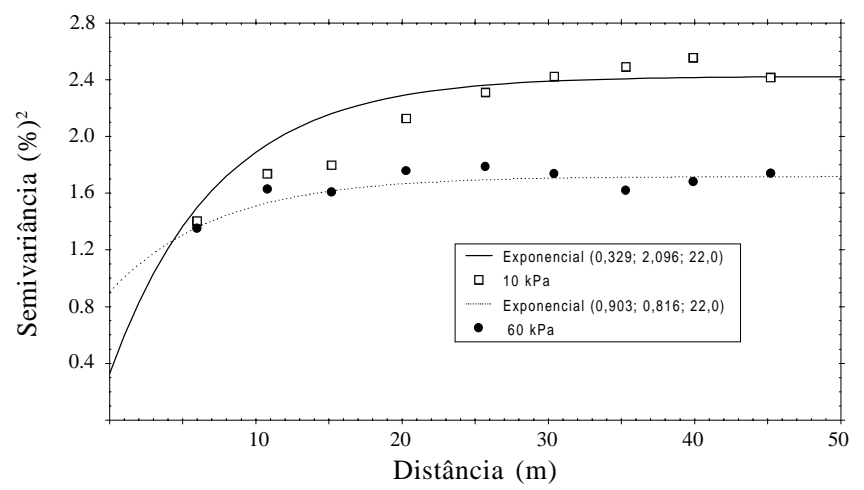

(A)

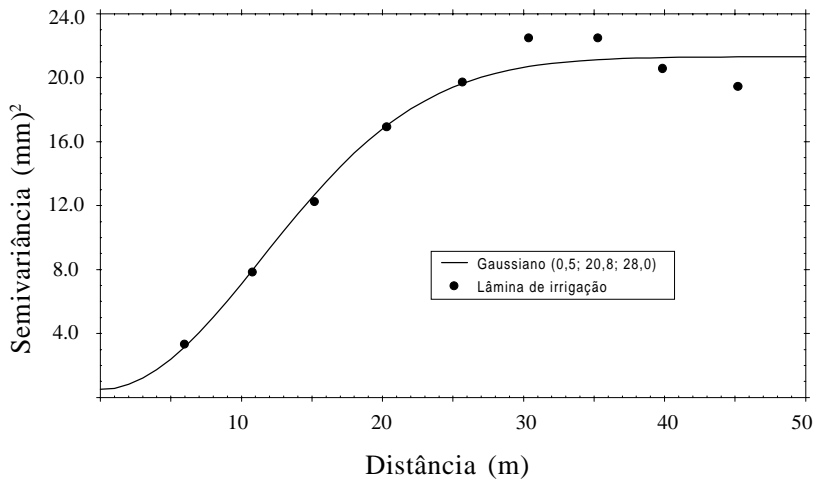

(B)

Figura 1. Semivariogramas experimentais e modelos ajustados para os dados de umidade volumétrica nas tensões de 10 e 60 $\mathrm{kPa}(\mathrm{A})$ e de lâmina aplicada (B)

Tabela 2. Parâmetros dos modelos ajustados aos semivariogramas experimentais dos dados de umidade volumétrica nas tensões de $10\left(\theta_{10}\right), 60 \mathrm{kPa}\left(\theta_{60}\right)$ e de lâmina aplicada

\begin{tabular}{lccccc}
\hline Modelo & $\begin{array}{c}\text { Efeito pepita } \\
\left(\mathrm{C}_{0}\right)\end{array}$ & $\begin{array}{c}\text { Patamar } \\
\left(\mathrm{C}_{0}+\mathrm{C}_{1}\right)\end{array}$ & $\begin{array}{c}\text { Alcance } \\
(\mathrm{m})\end{array}$ & $\begin{array}{c}\mathrm{C}_{\mathrm{o}} \\
\left(\mathrm{C}_{\mathrm{o}}+\mathrm{C}_{1}\right)\end{array}$ & $\mathrm{Vr}^{*}$ \\
\hline Exponencial & 0,329 & 2,435 & 22,0 & 0,14 & 1,001 \\
Exponencial & 0,903 & 0,816 & 22,0 & 0,52 & 1,003 \\
Gaussiano & 0,500 & 20,8 & 28,0 & 0,02 & 1,001 \\
\hline
\end{tabular}

Os dados de lâmina real necessária, apesar de não se ajustarem à normalidade, apresentaram distribuição relativamente simétrica, evidenciada pela proximidade dos valores da média e mediana (Tabela 1). Apresentaram, também, uma forte concentração de valores próximo à média, que se refletiu na pequena amplitude interquartílica e no alto coeficiente de curtose.

Os valores de lâmina aplicada não se ajustaram à distribuição normal e apresentaram assimetria negativa (Tabela 1). O coeficiente de uniformidade de Christiansen, do ensaio de irrigação, foi de $73 \%$, índice que pode ser considerado satisfatório, pois sistemas autopropelidos geralmente proporcionam baixa uniformidade de aplicação (Frizzone, 1992). 
A eficiência de armazenamento e os índices de drenagem profunda determinados pelo modelo linear, ficaram próximo dos obtidos, considerando-se a variabilidade do armazenamento de água no solo (Fig. 2A e B); este resultado pode ser atribuído à distribuição relativamente simétrica e à forte concentração dos dados de lâmina real necessária $\left(\mathrm{y}_{\mathrm{r}}\right)$; no entanto, o modelo linear foi menos sensível em diferenciar áreas deficiente e adequadamente irrigadas (Fig. 2C). Considerando-se uma irrigação com lâmina média aplicada $\left(\mathrm{y}_{\mathrm{m}}\right) 25 \%$ maior que $\mathrm{y}_{\mathrm{r}}\left(=1-\mathrm{y}_{\mathrm{r}} / \mathrm{y}_{\mathrm{m}}=0,20\right)$, o grau de adequação foi de $79 \%$. Para as mesmas condições, o valor obtido pelo modelo linear foi de apenas $67 \%$, comportamento que se deve ao fato do modelo linear assumir que a distribuição de freqüência das lâminas é totalmente simétrica. Com base no modelo linear, quando os valores de $\mathrm{y}_{\mathrm{r}}$ e $\mathrm{y}_{\mathrm{m}}$ forem iguais, $50 \%$ da área serão subirrigados e $50 \%$ serão irrigados adequadamente, condição ideal que dificilmente ocorre na prática, devido à distribuição das lâminas apresentar, geralmente, assimetria.

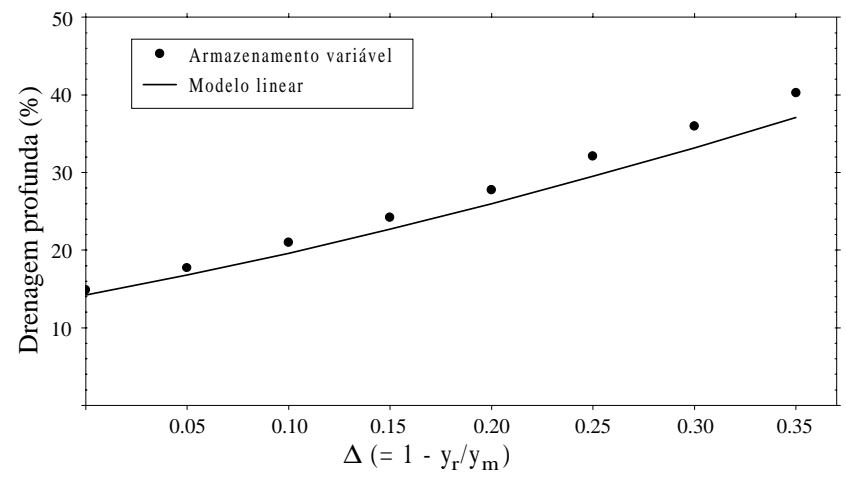

(A)

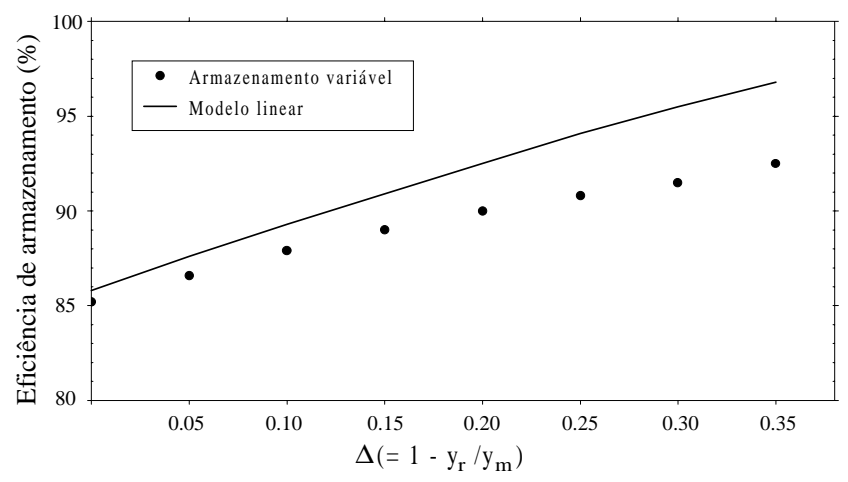

(B)

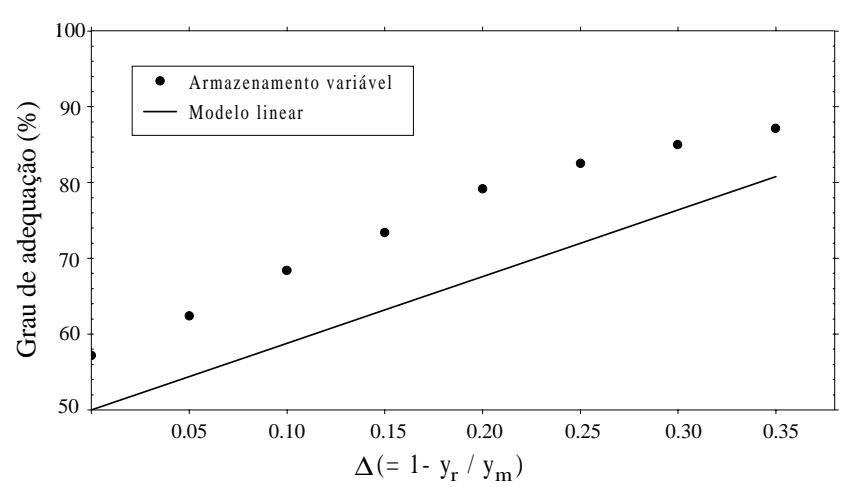

(C)

Figura 2. Drenagem profunda (A), eficiência de armazenamento (B) e grau de adequação (C) para diferentes relações entre lâmina real necessária $\left(\mathrm{y}_{\mathrm{r}}\right)$ e lâmina média aplicada $\left(\mathrm{y}_{\mathrm{m}}\right)$

\section{CONCLUSÕES}

1. Os valores de umidade nas tensões de 10 e $60 \mathrm{kPa}$ e de lâmina aplicada apresentaram dependência espacial.

2. A variabilidade do armazenamento de água no solo não influenciou, de forma relevante, a eficiência de armazenamento nem os índices de drenagem profunda.

3. O efeito da variabilidade foi maior na avaliação do grau de adequação, parâmetro subestimado pelo modelo linear.

\section{REFERÊNCIAS BIBLIOGRÁFICAS}

COMISSÃO CENTRO-SUL BRASILEIRA DE PESQUISA DE TRIGO. Recomendações para 1995. In: REUNIÃO DA COMISSÃOCENTRO-SUL BRASILEIRADEPESQUISADE TRIGO, 11., Cascavel, 1995. Cascavel: OCEPAR, 1995. 203p. CUENCA, R.H. Irrigation system design: An engineering approach. New Jersey: Prentice Hall, Englewood Cliffs, 1989. $552 \mathrm{p}$.

DOORENBOS, J.; KASSAM, A.H. Efectos del água sobre el rendimento de los cultivos. Roma: FAO, 1979. 212 p.

EMBRAPA. Centro Nacional de Pesquisa de Solos (Rio de Janeiro, RJ). Manual de métodos de análise de solo. 2. ed. Rio de Janeiro: 1997. 212p. EMBRAPA/CNPS. Documento, 1

ENGLUND, E.; SPARKS, A. Geo-EAS (Geoestatistical Environmental Assessment Software). Las Vegas: U.S. Environmental Protection Agency, 1988. 1. v.

FIETZ, C.R. Variabilidade espacial do armazenamento de água no solo visando ao manejo da irrigação por aspersão. Piracicaba: ESALQ/USP, 1998. 97p. Tese Doutorado

FRIZZONE, J.A. Irrigação por aspersão: Uniformidade e eficiência. Piracicaba: ESALQ/USP, 1992.53p.

FRIZZONE, J.A. Uniformidade e eficiência da irrigação. Piracicaba: ESALQ/USP, 1998. 40p.

ISAAKS, H.E.; SRIVASTAVA, R.M. An introduction to applied geostatistics. New York: Oxford University Press, 1989. 560 p.

KARMELI, D. Estimating sprinkler distribution patterns using linear regression. Transactions of the ASAE, St. Joseph, v. 21, n. 4, p. 682-686, 1978.

LEME, E.J.A. Avaliação da uniformidade e eficiência da irrigação através dos modelos normal e linear. ITEM- Irrigação e Tecnologia Moderna, Brasília, n. 27, p.11-14, 1986.

LIBARDI, P.L.; MANFRON, P.A.; MORAES, S.O.; TUON, R.L. Gravimetric soil water content variability of a hydromorphic soil. Revista Brasileira de Ciência do Solo, Campinas, v. 20, n.1, p. 1-12, 1996.

SOUZA, F. de; RIBEIRO, J.M. Aplicação do modelo linear de Karmeli na avaliação do sistema de irrigação por aspersão. ITEM - Irrigação e Tecnologia Moderna, Brasília, n. 16, p.7-10, 1984.

van GENUCHTEN, M.T. A closed-form equation for predicting the hydraulic conductivity of unsaturated soils. Soil Science Society of America Journal, Madison, v. 44, n. 5, p. 892-898, 1980.

VIEIRA, S.R.; HATFIELD, J.L.; NIELSEN, D.R.; BIGGAR, J.W. Geostatistical theory and application to variability of some agronomical properties. Hilgardia, Berkeley, v. 51, n.3, p. 1-75, 1983.

WALKER, W.R. Explicit sprinkler irrigation uniformity: efficiency model. Journal of the Irrigation and Drainage Division, New York, n. IR, p.129-136, 1979. 